\title{
Comparison of participant information and informed consent forms of five European studies in genetic isolated populations
}

\author{
Deborah Mascalzoni ${ }^{\star, 1}$, A Cecile JW Janssens ${ }^{2}$, Alison Stewart ${ }^{3}$, Peter Pramstaller ${ }^{1}$, Ulf Gyllensten ${ }^{4}$, \\ Igor Rudan $^{5,6}$, Cornelia M van Duijn ${ }^{2}$, James F Wilson ${ }^{6}$, Harry Campbell ${ }^{6}$ and Ruth Mc Quillan ${ }^{6}$ \\ on behalf of the EUROSPAN consortium
}

\begin{abstract}
Family-based research in genetically isolated populations is an effective approach for identifying loci influencing variation in disease traits. In common with all studies in humans, those in genetically isolated populations need ethical approval; however, existing ethical frameworks may be inadequate to protect participant privacy and confidentiality and to address participants' information needs in such populations. Using the ethical-legal guidelines of the Council for International Organizations of Medical Sciences (CIOMS) as a template, we compared the participant information leaflets and consent forms of studies in five European genetically isolated populations to identify additional information that should be incorporated into information leaflets and consent forms to guarantee satisfactorily informed consent. We highlight the additional information that participants require on the research purpose and the reasons why their population was chosen; on the potential risks and benefits of participation; on the opportunities for benefit sharing; on privacy; on the withdrawal of consent and on the disclosure of genetic data. This research raises some important issues that should be addressed properly and identifies relevant types of information that should be incorporated into information leaflets for this type of study.
\end{abstract}

European Journal of Human Genetics (2010) 18, 296-302; doi:10.1038/ejhg.2009.155; published online 14 October 2009

Keywords: informed consent; isolates; participation; EUROSPAN; information leaflets; ethics

\section{INTRODUCTION}

Family-based genetic research in isolated populations is an effective methodology for identifying loci influencing variation in disease traits. ${ }^{1,2}$ This approach has been used frequently to investigate genetic mutations for specific hereditary disorders such as familial forms of cancer and heart disease. Now, family-based study designs are also being used on a much larger scale to investigate genetic predisposition to diseases that have hitherto not been recognised as running in families. As this approach is not as widely used at present as large population-based studies, discussion of the ethical issues inherent in genetic epidemiological research has largely focused on genomics and biobanks $s^{3-8}$ and specifically on the tension between the promise of important advances in biomedical research ${ }^{6,9}$ and the requirement for robust ethical standards. ${ }^{70-12}$ New research techniques raise important ethical issues relating to consent ${ }^{13-16}$ ownership, governance, access to information, benefit sharing and community participation. These issues apply partly to every kind of genetic study, but although these issues are also applicable to family-based studies performed in genetically isolated communities, the special characteristics of such communities provide additional challenges to ethical approaches designed for large population-based biobanks. For example, safeguards designed to preserve participant privacy and confidentiality in large population-based studies may be inadequate in small, isolated communities. ${ }^{1718}$ Within the context of family-based research in a small community, standard procedures for anonymising participant information ${ }^{19-21}$ may be inadequate for protecting the identities of families. Furthermore, research in these isolated populations must also address additional ethical issues. For instance, communities are selected because they are genetically more homogeneous than nonisolate populations, a criterion that is itself loaded with emotive associations. ${ }^{22}$ On the one hand, such communities may be regarded or may regard themselves as special or unique: on the other, there may be derogatory associations with inbreeding or those being cutoff from the world. These issues are of particular concern when the population and/or specific pedigrees are described in scientific publications. As in other genetic studies, family-based studies conducted in genetically isolated populations can potentially uncover information about a family's health that may have wider implications for individual members of that family, even if the individuals concerned were nonparticipants. This is especially relevant when family trees are drowned and where information about ancestries is collected. Those issues need to be clearly communicated so that both families and individuals

\footnotetext{
${ }^{1}$ Institute of Genetic Medicine, European Academy Bozen/Bolzano (EURAC), Bolzano, Italy; ${ }^{2}$ Department of Epidemiology, Erasmus MC, University Medical Center Rotterdam, Rotterdam, The Netherlands; ${ }^{3}$ PHG Foundation, Worts Causeway, Cambridge, UK; ${ }^{4}$ Department of Genetics and Pathology, Rudbeck Laboratory, Uppsala University, Uppsala, Sweden; ${ }^{5}$ Faculty of Medicine, Croatian Centre for Global Health, University of Split, Split, Croatia; ${ }^{6}$ Centre for Population Health Sciences, Medical School, University of Edinburgh, Edinburgh, UK

*Correspondence: Dr D Mascalzoni, Institute of Genetic Medicine, EURAC Research, Viale Druso 1, Bolzano I-39100, Italy. Tel: +39 471 055513; Fax: +39 471 055599; E-mail: deborah.mascalzoni@eurac.edu
}

Received 15 December 2008; revised 24 August 2009; accepted 28 August 2009; published online 14 October 2009 
can make satisfactorily ${ }^{23}$ informed decisions about participation. Several ethical-legal guidelines are available that specify the kinds of information that should be provided in participant information leaflets and informed consent forms to ensure that participants are able to give satisfactorily informed consent. Examples include the general guidelines of the Council for International Organizations of Medical Sciences (CIOMS), and the guidelines of the National Office of Public Health Genomics/Centers for Disease Control and Prevention (CDC) and the Public Population Project in Genomics (P3G) that specifically focus on genetic studies. ${ }^{24-26}$ Such guidelines are in addition to national and local research ethics guidelines and legislation, which provide a roadmap for researchers compiling participant information and consent forms. Yet, such guidelines tend to be very general in scope and do not address the specific issues that apply to genetically isolated populations.

The aim of this paper is to identify specific issues that should be addressed and incorporated into participant information leaflets and consent forms for family-based studies in genetically isolated populations. All the authors are working on different levels in genomic studies in isolates, and although all the information leaflets and consent forms for the five studies comply with national research ethics requirements, they differ in many aspects. This led us to this work. Taking the comprehensive CIOMS checklist of the international guidelines (CIOMS $\mathrm{G})^{25}$ as a starting point, we identified the topics that, from our direct experience, needed to be addressed differently in genetic isolates. Next, we compared the participant information and informed consent forms of five European studies in genetically isolated populations to identify what additionally needed to be addressed.

\section{METHODS}

We used the International Ethical Guidelines for Biomedical Research Involving Human Subjects of the CIOMS ${ }^{25}$ to examine the participant information of studies in genetically isolated populations (see Supplementary information). We reviewed the criteria one by one, in each case examining whether the topic raises new concerns specific to family-based studies in isolates. We compared the participant information and informed consent forms of five studies participating in the EUROpean Special Populations reseArch Network (EUROSPAN). The studies are summarised briefly here, with further information in Supplementary Appendix 1.

The Erasmus Rucphen Family study ${ }^{27}$ (ERF; the Netherlands) is a familybased study that investigates the genetic origins of complex diseases in a genetically isolated community in the south-west of the Netherlands. The study population essentially consists of one extended family of descendants from 20 related couples that lived in the isolate between 1850 and 1900. All descendants were ascertained, and descendants of age 18 years and older were invited to participate. All participants underwent medical examinations and completed several questionnaires.

MICROisolates in South Tirol (MICROS; Italy) ${ }^{28}$ is a family-based population study that investigates the genetic origins of complex diseases. The participants in the villages were selected on the basis of four criteria for isolation: (i) evidence of an old settlement; (ii) a small number of founders; (iii) high endogamy rates; (iv) slow or null population expansion and negligible immigration. The expression 'microisolate' was used to distinguish small subpopulations in remote, high valleys that have conserved an even higher degree of isolation than the cultural and linguistic South Tyrolean isolate.

The Orkney Complex Disease Study (ORCADES; UK) is a family-based study that seeks to identify the genetic factors influencing cardiovascular and other disease risks in the population isolate of Orkney, an archipelago off the north coast of Scotland. Individuals aged 16 years and over and with at least one grandparent from the remote North Isles of Orkney were invited to participate. As above, all participants underwent a variety of medical examinations and provided information on health, lifestyle and family history of disease.
The Northern Sweden Population Health Study (NSPHS; Sweden) is a family-based population study that aims to identify genetic and environmental risk factors for common, mainly non-communicable disease in populations living in the arctic and sub-arctic areas. The study includes one population in the southern part of the Swedish mountain range and one population located in the extreme far north of Sweden on the border with Finland. All inhabitants over the age of 15 were invited to participate. About $60 \%$ of those invited $(740$ individuals) participated in the study and underwent a comprehensive health examination and sampling for serum, plasma and DNA analyses. Information on genealogy, lifestyle, diseases and diet was collected.

The Croatian study (CROAS; Croatia) ${ }^{29,30}$ is a family-based study of residents of small villages in a Dalmatian island. The village populations of this and neighbouring islands in Croatia represent a well-characterised meta-population of genetic isolates. The CROAS study investigated approximately 1000 unselected Croatian residents, aged 18-93 years, from villages on the Dalmatian island. As above, each participant was examined for a large number of disease-related traits and serum, plasma and DNA samples were obtained.

\section{RESULTS}

Table 1 lists the topics from the CIOMS G that may raise additional information requirements for family-based studies in isolated populations. The extent to which the participant information leaflets and informed consent forms of the five studies already address these issues is summarised below.

\section{Selection of population}

Item 1 of the CIOMS G states that individuals should be informed about why they have been selected as suitable for the research. The CIOMS G is silent on the selection of the population, as opposed to the individual; however, for research in isolated communities, this is a key issue. It is incumbent on researchers to explain the reason why the population was chosen for the research, particularly when the selection criterion is made explicit in future publications. Furthermore, the population should be described in publications in a way that is not stigmatising or in any other way harmful to participants or other members of the community. Only MICROS and ORCADES explicitly specify that the communities were selected for being more genetically and environmentally homogeneous than the general population. ORCADES contrasts the Orkney isles with urban populations, where there is 'a variety of occupations, lifestyles and ethnic backgrounds', but is not explicit about the scientific reasons for choosing the population. MICROS explains that historical studies were carried out to identify suitable research populations and explicitly states that the criteria used were high levels of endogamy and inbreeding, low levels of immigration and the availability of good historical information. ERF states that participants were invited if they were descended from 20 couples living in the village between 1850 and 1900; however, the reason why the descendants of these couples were of interest is not made explicit. ORCADES and ERF do not assess all inhabitants of the community and both specify that only a selection was invited, but they do not specify why that selection was made. NSPHS states that the study aims to investigate the health of those living in the arctic and sub-arctic regions, giving both environmental and historical reasons for population choice: settlements of long standing with stable populations, little immigration and traditional lifestyles were chosen.

\section{Consent to participate}

Informed consent is a requirement for every research conducted on human subjects, based on the notion of autonomy of the subject who has the right to decide. An intervention in the health field may only be 
Table 1 Review of the appropriateness of Council for International Organizations of Medical Sciences (CIOMS) guidelines checklist (G): additional concerns that apply to family-based studies in isolated populations

CIOMS G

Key issues and additional informational requirements for family-based studies in genetically isolated populations.

Selection of population (G1)

Defining a population as an isolate could be stigmatising if not defined clearly.

There may be a mismatch between the way in which a population is described in participant information leaflets and the way it is subsequently described in scientific publications.

The reasons that the population is considered suitable for the research should be made explicit and this information should be consistent between the participant information sheet and any future publications.

Withdrawal of consent (G2)

There may be technical problems in withdrawing all data on a single individual.

Withdrawal of single individuals may have consequences for collected family data.

Participants may feel under additional pressure from family members to participate or not participate in the study.

The right to withdraw from the study can only be meaningful if people are updated about major changes in the direction of the project.

Researchers should make clear what will happen to the data relating to withdrawn participants, including genealogical data.

Information leaflets should state clearly that participation is an individual decision but also that this may have implications for the rest of the biological family.

Major changes in project direction should be communicated to participants so that they are able to re-evaluate their decision to participate.

Research purpose (G3)

Participants may not be aware that researchers routinely verify pedigree relationships using genetic information. Crucially, this could potentially provide researchers with information, which may contradict that provided directly by the participants - for example, on paternity.

It should be made clear that the research is on extended families. The implications of this should be clearly explained: this means that family trees will be reconstructed and that pedigree relationships will be verified using genetic information.

Benefit sharing (6)

There is greater potential to direct benefits to the study population and to involve the population in decisions about benefit sharing because the study populations are small and well defined, in contrast to large, dispersed, population-based studies.

Researchers should engage with the community regarding the types of benefits that might accrue and those that would be welcomed.

Potential risks of (G9) participation

There are potential informational risks to participants' family members and to the wider community, whether or not they are themselves participants.

The potential risks to participants, their families and the wider community should be clearly explained, including learning about family illnesses, increased insurance premiums, disclosure of non-paternity, community anxiety about specific disease risk, and the use of data for criminal investigations.

Expected benefits of research (G11)

Research projects in small, isolated communities typically have a high profile, which may easily lead to unrealistic expectations as to what the study might find and what benefits might flow from this.

Care must be taken not to make unrealistic claims about the benefits likely to accrue to the community from the study.

Privacy concerns (G14)

Standard safeguards are insufficient to guarantee privacy, not just for the single individual but for the whole biological family. Through combined data (genealogies, health data, genotypes), families and individuals in the family may be identifiable.

There is a need to be open about the difficulties of guaranteeing complete privacy and to ensure that if a publication might potentially identify individuals and families, fully informed consent is obtained from those individuals and families before publication.

Disclosure of genetic data (G16)

Linked genetic, genealogical and health data can provide information about all members of the biological group, including non-participants.

There may be legal requirements to disclose data to the police, courts or other third parties in certain circumstances.

Families and individuals are more easily identifiable in small, close-knit communities than in more anonymous populations.

Researchers must state under what circumstances genetic information will be shared with external agencies (research partners, official agencies).

Information sheets should clearly state the policy according to the current law, for example, that data will not be disclosed to police/court unless there is a court order. In publishing study findings, researchers must take great care not inadvertently to identify families through indirect information ('the family in the study village with three affected children...').

carried out after the person concerned has given free and informed consent to it. As defined by the Council of Europe, ${ }^{31}$ informed consent is ensured through appropriate information about the purpose and nature of the intervention as well as on its consequences, benefits and risks. This freedom does not end with the provision of consent and, in fact, the person concerned may freely withdraw consent at any time. In genomics studies in general, it is very difficult to foresee every possible development of research. This means that the consent to participate in 
a project based on transparent and complete information needs to be implemented on the basis of this fact. For this reason, many of the points raised in the discussion aim at ensuring a proper and transparent communication with the participants to allow a free choice.

\section{Withdrawal of consent}

The right to withdraw (CIOMS G article 2) is enshrined in every type of research on human participants. ${ }^{25,31}$ It is, however, a particularly sensitive issue in this context, entwined as it is with issues of privacy and confidentiality, not just for the individual participant but also for non-participating relatives, as genetic information is shared by the biological pedigree. So first, in a small community, participants might feel pressurised either to participate or not to participate by family members or others in the community. The key issue, however, is the lack of clarity about what withdrawal of study participation actually means, especially when the family as a whole is an essential source of information in family-based studies. Withdrawal can refer to the destruction of personal information, destruction of the whole set of data about an individual including anonymised data, or the destruction of the specimens as well. ${ }^{17,32}$ The precise details of what will happen to the data and samples of participants who have withdrawn from the study are often not specified. In practical terms, anonymised data or shared data ${ }^{16}$ may be difficult or impossible to destroy, and data that have already been analysed are not completely destroyable, especially if linked in a characterised genealogical tree. ${ }^{12,33}$ If, for these reasons, complete withdrawal from the study is not possible, this should be made explicit at the outset. Finally, the right to withdraw consent can only be meaningful if participants are kept up-to-date about any changes in the purpose or direction of the study. The only leaflet to comment explicitly on potential family or community pressure on individuals to participate is the ERF one, which states that participation is an individual decision and that individuals should not feel guilty towards their relatives because of the decision they make. Regarding participant withdrawal, all studies except CROAS state that participants are free to withdraw at any time without giving any reason. ORCADES and CROAS give no information on what happens to data and samples if a participant withdraws. MICROS states that data will either be removed from the study or completely anonymised, where removal is not possible (as, for instance, in shared data), and samples destroyed. ERF states that data and biological samples will be removed. The NSPHS study states that withdrawal implies destruction of the samples, but is unclear about what happens to data in these circumstances. None of the studies mentions what happens to genealogical data if participants withdraw.

\section{Research purpose}

Item 3 of the CIOMS G specifies that individuals should be informed about the purpose of the research. Genetic studies in isolated communities aim to unravel the contribution of genetic factors in common diseases, but in contrast to population-based cohort studies, they do this using genealogy data. We agree that participants should be informed that family trees will be reconstructed on the basis of genealogy data, which implies that pedigree relationships can be verified using genetic data, but that data on genealogies will neither be shared nor disclosed. Crucially, participants must be informed that this provides researchers with information that may contradict that provided by the participants themselves (eg, on paternity). ORCADES describes 'tracking the inheritance of genes in families' but is not explicit about tracing family trees. CROAS and ORCADES do not mention family tree reconstruction, although this is clearly implied, as a genealogical form is attached for participants to complete. MICROS and ERF state explicitly that family trees are reconstructed, but neither explains that this might potentially reveal non-paternity or how such sensitive information will be treated.

\section{Benefit sharing}

The CIOMS states that researchers must provide information on whether money or other remuneration will be provided in return for the individual's participation (item 6) and on whether participants will benefit from any commercial products developed as a result of the research (item 20). Again, the CIOMS G is explicit only about sharing benefits with individuals, not communities; however research in nonisolate populations offers the potential to direct benefits to the study population and to involve the population in decisions about benefit sharing. Although few if any commercial benefits are anticipated as a result of this type of study, the fact that these studies are located in small, well-defined communities means that were commercial benefits to accrue, and that it would be much easier to share the proceeds with the community than it would be in a bigger study. Accurate information about the potential financial gains and transparency about who will benefit is essential to create realistic expectations. ${ }^{3,11,24,34,35}$ Many studies show that participants are generally interested in knowing this information; however, this would not necessarily make a difference to their decision to participate. ${ }^{36,37}$

The MICROS information leaflet states that although no commercial benefits are anticipated, any that are forthcoming will be reinvested in research and health facilities in the region but that there will be no individual benefits to participants. The CROAS information leaflet mentions the possibility of the commercial use of study results but does not mention benefit sharing or financial benefit to the community. The ERF information sheet does not mention benefit sharing. The ORCADES consent form explicitly states that participants will not benefit financially if the research leads to the development of new treatments or medical tests, but no mention is made of community financial benefit. In the NSPHS study, although commercial applications were discussed at community information meetings, this subject was not included in the informed consent form.

\section{Potential risks of participation}

Article 9 of the CIOMS G specifies that participants be informed about any foreseeable risks, pain, discomfort or inconvenience arising from participation, including risks to the participant's spouse or partner. In the context of family-based research in isolated populations, there are potentially significant informational risks to participants' family members and to the wider community - including nonparticipants. These include learning about family illnesses, increased insurance premiums, disclosure of non-paternity and community anxiety about specific disease risks.

The CROAS study does not mention any potential risks. MICROS, ORCADES and ERF mention only the potential physical risks of participation (eg, bruising at the site of venepuncture), but no risks associated with unanticipated information. NSPHS discusses the risks with the community before the start of the study but does not go into detail in the information leaflet. None of the information leaflets identifies misuse of data, non-paternity disclosure, community stigmatisation or family conflict as potential risks, despite the fact that pedigrees and individual health and genotype data are held and used.

\section{Expected benefits of research}

Article 11 of CIOMS G specifies that participants should be informed about the expected benefits of the research to the community or 
society in general or to scientific knowledge. The expected benefits to society are generally indirect, as this kind of research does not provide new cures or results that are of immediate use to the population. In small, isolated communities, where the research project has a high profile, it is important not to create false expectations as to what the study might find and what benefits might flow from it. MICROS makes a very general statement about the possible future health benefits following from new knowledge gained through the study. CROAS and ORCADES imply a benefit to the local community, stating that the study may uncover information that may improve the community's health. ORCADES, NSPHS and ERF mention the thorough health examination as an individual benefit, with the potential of alerting participants' general practitioners about unexpected incidental findings. CROAS suggests that the project may improve the health of the island but does not state how this might happen. In general, the information leaflets take a very narrow view of the potential benefits to participants, when in fact this type of study can have many very positive, if less obvious, spin-offs for participants. NHPHS and ORCADES, for example, have conducted various meetings and lectures with a health promotion/educational focus and ERF has conducted annual information meetings to inform general practitioners about the preliminary results. These approaches have been valuable in building trust between the participants and the scientists, in addition to the health promotion benefits. ERF participants have received several information leaflets with information about the study progress and also been invited to an information meeting at the end of the data collection period. NHPHS has found that focusing on the village has provided a boost to the community, enabling them to see themselves as part of a larger European project. In south Tyrol, the publication of two historical books about genetics and the history of the population proved a very popular way of feeding back some of the results to participants. For some, participation has also led to the early diagnosis of medical problems.

\section{Privacy concerns}

The CIOMS G specifies that participants must be informed about the arrangements for safeguarding their privacy and the confidentiality of records (article 14). The privacy of individual genetic data is a key concern ${ }^{19,33,38,39}$ because of the potential discriminatory consequences of misuse of such data by insurance companies, health-care providers and so on (examples of this misuse may be uninsurability, or having to pay a higher price if carriers of certain gene $\operatorname{etc}^{20}$ ). Even if data are not directly identifiable, the possibility of cross-matching data with individuals cannot be ignored: in small, isolated communities, standard: safeguards may be insufficient to guarantee privacy, both for individual participants and for their families. Unusual families may be identifiable through a combination of genealogical, genetic and health data. Although in some communities the genealogical information is in the public domain, such data must be regarded as personal information under the European Privacy Law, particularly in the scientific research context, where genealogical data are systematically collected by professional researchers and cross-linked with medical and genetic information. The resulting data may be very different from socially known genealogies, raising issues of data confidentiality and security as well as presenting practical difficulties for participant withdrawal as described above. The release of such highly sensitive information may be disruptive for a small community, with the potential of uncovering information on, for example, false paternities and undisclosed adoptions. Enhanced genealogies should, therefore, be stored securely and confidentially. Although all countries have strict laws governing the handling and storage of personal data and these issues were often discussed in meetings with the communities, they tend not to be addressed at length in the information leaflets. The MICROS information leaflet states that data will be handled in accordance with the Italian Privacy law, which also means the separation of health data and genealogies from personal data. The only information leaflet to mention the risk of identification of participants in publications is that of the ORCADES study and this states that individuals will not be identifiable in any way in research publications; however, this may be a problematic point as it is hard to guarantee this $100 \%$ in studies of this kind.

\section{Disclosure of genetic data}

Article 16 of the CIOMS G states that researchers must inform participants about their policies regarding the disclosure of personal and familial genetic data to third parties. This is important because in family-based genetic research, genealogies linked to genetic data may contain information about non-participants, which may be useful to a range of external agencies and which researchers may be legally obliged to hand over in certain situations. For example, in the UK, data must be disclosed if there is a court order authorising this (as in the case of crimes, even minor ones, or if there is the need to assess facts relevant to public security). Article 16 also has implications for the publication of research findings in scientific journals. In small, close-knit communities, individuals with genetic conditions can potentially be identified from very little information ('the family in the study village with three affected children...') and information can spread very quickly and affect family life more strongly than in more anonymous environments. Publication policies for this type of study must, therefore, first ensure that individual and family identities are anonymised wherever possible, and second, that where this is not possible, consent to publication is obtained from the individuals and families concerned.

Although most of the information leaflets state that data will be shared only with research partners or collaborators, these terms are not clearly defined and neither are the arrangements for the safe exchange of data made explicit. ${ }^{40}$ The MICROS and ERF studies state that anonymised data will be shared with research partners for specific research purposes only. The ERF leaflet explains that research partners will only have access to coded data. The CROAS information leaflet mentions that data will not be shared with anyone outside the research team. In the meetings before the initiation of the NSPHS study, it was made clear that the study was part of a wider collaboration; however, this information is not included in the information leaflet. ORCADES states that data will not be made available to any other authorities. None of the studies mentions police/court access to data, which is an issue in some but not all countries (Italy's strict privacy laws, eg, mean that such data cannot be obtained by the courts).

Table 1 summarises what we regard as essential information to ensure adequate informed consent for family-based studies in isolates.

\section{DISCUSSION}

We reviewed the information and informed consent forms of five family-based studies in genetically isolated populations following the CIOMS G and showed that the existing guidelines may not be fully appropriate for these types of studies to deal with all the issues that are relevant to this kind of studies. Before discussing the implications in more detail, two points need to be addressed. First, it is important to emphasise that the information leaflets and consent forms of all five studies complied fully with national research ethics requirements in the five nations concerned. This review highlights additional issues specific to family-based genetic studies in isolated populations that we 
feel are not adequately addressed by the existing national and European ethics guidelines. These are raised with the aim of trying to improve recognised best practice and international guidelines for the conduct of these studies. Our review of the CIOMS checklist selects only those topics that may raise additional issues specific to this type of study. We did not compare generic topics that are relevant to all genetic medical studies ${ }^{10,12,41}$ (eg, information on the funding of the research, the ethical review process, disclosure of data in society and genetic discrimination). ${ }^{7,10,41}$ Second, most topics that were not addressed in the information leaflets may have been communicated in other ways by the studies, for example, in information meetings and brochures and in face-to-face discussions between individual participants and research staff. This greatly helped provide transparent and careful information and was greatly appreciated by the communities. This also highlights the central importance of other forms of communication with participants and communities.

Several interesting points follow from our comparison. First, we observed that there is often a discrepancy between the research purpose as it is described in scientific publications and how this is communicated to potential participants in information and consent documentation. In general, there was scant explanation as to why these specific populations were invited to participate. Hardly any of the studies mentioned the term 'genetically isolated population' or referred to endogamy or inbreeding, and yet these descriptions are used by researchers in scientific publications. These are highly sensitive issues that could potentially affect the communities concerned. To some extent, discrepancies between the way research aims are communicated to participants and to the scientific community may be attributable to the difficulties in communicating complex scientific concepts to a lay audience; to some extent, they reflect the fluidity of research aims that often evolve over time in a fast-moving field. It is obviously important, however, to ensure that these discrepancies do not arise because of the researcher's fear that providing full information on research aims might have a detrimental effect on participation rates.

Second, when addressing the implications of participation, most studies identified minor physical consequences or potential benefits of having a personal health check. Little or no emphasis, however, was given to the potentially more significant consequences of participation, most notably the possible informational implications, both positive and negative, for both individuals and families, such as discovering new information about family health, especially in an easily identifiable community. ${ }^{15,20,42-44}$ It may be that participant information leaflets are not an adequate means of addressing this type of information, but if this is the case, it raises the question as to in what way and at what stage of the process this issue should be addressed.

Third, all studies mentioned that participants' personal information is stored under a code that provides a reasonable guarantee of not being traceable back to personal data. Complex family trees are not, however, completely anonymous and can lead to the dissemination of personal and family health information of an extremely sensitive nature if cross-matched with other data, for example, when presented in scientific publications. Clearly, researchers should be aware that family trees could be recognised if steps are not taken to anonymise them and that this has consequences for the confidentiality of the accompanying medical information.

Fourth, the potential stigmatisation of communities is an issue that demands consideration in studies of this type. Perceptions of risk vary greatly between localities and this is reflected in different policy approaches, for example, regarding disclosure of information in publications. ${ }^{45,46}$ This is also the case in the EUROSPAN study populations: for example, the MICROS publication policy states that individual villages should not be named in any publication where this might result in stigmatisation (name of illness result related to specific villages), but can be named in more neutral publications (general description of the institute and of the scopes of research). Similarly, ORCADES publications do not identify individual isles, except in papers that are purely investigating the population sub-structure within Orkney. In the NSPHS study, participants and potential participants expressed a concern that single pedigrees should not be revealed or used in any publication, because of the risk of identifying specific families in such a small community. In contrast, a technical CROAS publication provides the inbreeding coefficient data of named villages. The ERF community name is included in the project's acronym, which is widely used in publications and elsewhere. In the internet era, accessing specialist publications across national borders is increasingly easy, and maintaining different publication policies may be problematic for international collaborators and could potentially raise serious issues of trust. For example, the failure to discuss the issue of inbreeding at the consent stage might make researchers vulnerable to a charge of lack of transparency if participants access publications whose focus is the inbreeding coefficient.

Fifth, this review has highlighted the importance of communication as a process that starts before the project commences, continues through the information and consent stage, and is ideally carried on throughout the project through to the dissemination of study findings. This is consistent with the findings of qualitative work on perception, which have found that study participants actively seek more information about research processes and results. ${ }^{47-49}$ Web pages, printed newsletters, regular meetings with participants or update letters to individuals are all useful means of keeping in touch, particularly in such a fast-moving research field. Keeping channels of communication open throughout the life of the project is clearly essential if satisfactorily informed consent is to be achieved. ${ }^{16,50}$

Finally, our research highlights the importance of the community, alongside the individual, in this type of research. Linking genealogical, health and genetic data in a small, isolated community provides researchers with information not just about individual participants but also about the whole biological group - participants and nonparticipants alike. The implications for and rights of non-participants in this type of study are dimensions that are missing in current research guidelines, but it is essential that researchers working in family-based genetic studies in isolated populations engage with the wider community so that non-participants are also aware of the possible implications of the study and given an opportunity to make their voices heard. It is important to reiterate that although we have concentrated fairly narrowly here on the information given to participants through the information and consent stage of the project, many of the issues raised here were addressed by all our projects at other stages, starting from discussions with research ethics committees at the project inception stage, through to public meetings and one-toone communications with individual participants later on. From this review, it is clear that generic guidelines cannot adequately address all the ethical issues of this type of study yet. Differences between studies and populations may require that specific topics be added to the information leaflet to ensure that participants have the information they need to make an informed decision. Nevertheless, we have identified a group of issues that merit special attention in all these types of study and should be addressed not only in the studies (special Information procedures, more comprehensive information leaflets and so on) but also on a normative level. International collaborations 
face additional challenges resulting from national differences in ethical norms and procedures, which can result in different standards and requirements. ${ }^{18}$ Therefore, there is a need for a harmonising approach for research in isolates that is equally applicable in different national contexts. The results of this analysis should not be seen as a definitive set of guidelines for family-based research in isolated communities but rather as a first step at raising some important issues that can then be taken forward in the public arena.

1 Peltonen L, Palotie A, Lange K: Use of population isolates for mapping complex traits. Nat Rev Genet 2000; 1: 182-190.

2 Shifman S, Darvasi A: The value of isolated populations. Nat Genet 2001; 28: 309-310.

3 Chadwick R, Berg K: Solidarity and equity: new ethical frameworks for genetic databases. Nat Rev Genet 2001; 2: 318-321.

4 Chadwick R, Schuklenk U: The politics of ethical consensus finding. Bioethics 2002; 16: iii-iiv.

5 Knoppers BM: Biobanking: international norms. J Law Med Ethics 2005; 33: 7-14.

6 Knoppers BM: Biobanks: simplifying consent. Nat Rev Genet 2004; 5: 485

7 Gibbons SM, Kaye J: Governing genetic databases: collection, storage and use. Kings Law J 2007; 18: 201-208.

8 Winickoff D: Governing population genomics: law, bioethics, and biopolitics in three case studies. Jurimetrics 2003; 43: 187-228.

9 Knoppers BM: Consent revisited: points to consider. Health Law Rev 2005; 13 : 33-38.

10 Foster MW, Sharp RR: Ethical issues in medical-sequencing research: implications of genotype-phenotype studies for individuals and populations. Hum Mol Genet 2006; 15 (Spec no. 1): R45-R49.

11 Hugo EC: Statement on pharmacogenomics (PGx): solidarity, equity and governance. Genomics, Society and Policy 2007; 3: 44-47.

12 Gibbons SMC, Kaye J, Smart A, Heeney C, Parker M: Governing genetic databases: challenges facing research regulation and practice. J Law Soc 2007; 34: 163-189.

13 Caulfield T, Ogbogu U, Nelson E et al: Stem cell research ethics: consensus statement on emerging issues. J Obstet Gynaecol Can 2007; 29: 843-848.

14 Caulfield T: SSRN-Biobanks and Blanket Consent: the Proper Place of the Public Good and Public Perception Rationales, 2008.

15 McGuire AL, Caulfield T, Cho MK: Research ethics and the challenge of whole-genome sequencing. Nat Rev Genet 2008; 9: 152-156.

16 Mascalzoni D, Hicks A, Pramstaller P, Wjst M: Informed consent in the genomics era. PLoS Med 2008; 5: e192.

17 Arnason V: Coding and consent: moral challenges of the database project in Iceland. Bioethics 2004; 18: 27-49.

18 Elger BS, Caplan AL: Consent and anonymization in research involving biobanks: differing terms and norms present serious barriers to an international framework. EMBO Rep 2006; 7: 661-666.

19 Lowrance WW, Collins FS: Ethics. Identifiability in genomic research. Science 2007; 317: 600-602.

20 Liberty: Inside Information. Balancing interests in the use of personal genetic data, Liberty, Response to Human Genetics Commission's 'Whose hands on your genes?', 2002.

21 UNESCO: International declaration on human genetic data, 2003.

22 Arnason E, Sigurgislason H, Benedikz E: Genetic homogeneity of Icelanders: fact or fiction? Nat Genet 2000; 25: 373-374.
23 Edwards J: Taking public understanding seriously. New Genet Soc 2002; 21: 315-325.

24 CIOMS: CIOMS guidelines 'Special ethical considerations for epidemiological research' Response from the Medical Research Council and the Wellcome Trust 15 December 2006, 2006; 4: 1.

25 CIOMS: International Ethical Guidelines for Biomedical Research Involving Human Subjects, 2002

26 WHO: Genetic Databases: Assessing the Benefits and the Impact on Human and Patient Rights, 2003.

27 Pardo LM, MacKay I, Oostra B, van Duijn CM, Aulchenko YS: The effect of genetic drift in a young genetically isolated population. Ann Hum Genet 2005; 69: 288-295.

28 Pattaro C, Marroni F, Riegler A et al: The genetic study of three population microisolates in South Tyrol (MICROS): study design and epidemiological perspectives. BMC Med Genet 2007; 8: 29.

29 Rudan I, Campbell H, Rudan P: Genetic epidemiological studies of eastern Adriatic Island isolates, Croatia: objective and strategies. Coll Antropol 1999; 23: 531-546.

30 Vitart V, Biloglav Z, Hayward C et al: 3000 years of solitude: extreme differentiation in the island isolates of Dalmatia, Croatia. Eur J Hum Genet 2006; 14: 478-487.

31 Europe Co: Convention for the Protection of Human Rights and Dignity of the Human Being with regard to the Application of Biology and Medicine: Convention on Human Rights and Biomedicine, 1997.

32 Helgesson G: The right to withdraw consent to research on biobank samples. Med Health Care Philos 2005; 8: 315-321.

33 Milanovich F, Pontille D, Cambon-Thomsen A: Biobanking and data sharing: a plurality of exchange regimes. Genomics, Society and Policy 2007; 3: 17-30.

34 HUGO EC: Statement of Benefit Sharing, 2000.

35 Merz F, McGee GE, Sankar P: Iceland Inc.? On the ethics of commercial population genomics. Soc Sci Med 2004; 58: 1201-1209.

36 Haddow G, Laurie G, Cunningham-Burley S, Hunter KG: Tackling community concerns about commercialisation and genetic research: a modest interdisciplinary proposal. Soc Sci Med 2007; 64: 272-282.

37 Kettis-Lindblad A, Ring L, Viberth E, Hansson MG: Genetic research and donation of tissue samples to biobanks. What do potential sample donors in the Swedish general public think? Eur J Public Health 2006; 16: 433-440.

38 Lunshof JE, Chadwick R, Vorhaus DB, Church GM: From genetic privacy to open consent. Nat Rev Genet 2008; 9: 406-411.

39 Malin BA, Sweeney L: A secure protocol to distribute unlinkable health data. AMIA Annu Symp Proc 2005; 485-489.

40 Kaye J, Heeney C, Hawkins N, de Vries J, Boddington P: Data sharing in genomics re-shaping scientific practice. Nat Rev Genet 2009; 10: 331-335.

41 Gulcher JR, Stefansson K: Ethics of population genomics research. Nature 1999; 400: 307-308.

42 Langlois A: The UNESCO Universal Declaration on Bioethics and Human Rights: perspectives from Kenya and South Africa. Health Care Anal 2008; 16: 39-51.

43 Rose H: An ethical dilemma. Nature 2003; 425: 123-124.

44 Rose $\mathrm{H}$ : The commodification of bioinformation: the Icelandic Health Sector Database, 2000.

45 Funtowicz SR: Science for the post-normal age. Futures 1993; 25: 735-755.

46 Petersen A: Biobanks 'engagements': engendering trust or engineering consent? Genomics, Society and Policy 2007; 3: 31-43.

47 Weill C: Can consultation of both, experts and the public help developing public policy? Science and Public Policy 2003; 30: 199-203.

48 Upshur RE, Lavery JV, Tindana PO: Taking tissue seriously means taking communities seriously. BMC Med Ethics 2007; 8: 11

49 Tutton R Corrigan editors: Genetic Databases: Socio-ethical Issues in the Collection and Use of DNA. London: Routledge, 2004.

50 Prainsack B, Reardon J, Hindmarsh R, Gottweis H, Naue U, Lunshof JE: Personal genomes: misdirected precaution. Nature 2008; 456: 34-35.

Supplementary Information accompanies the paper on European Journal of Human Genetics website (http://www.nature.com/ejhg) 